ITP-SB-93-63

November, 1993

\title{
General Determination of Phases in Leptonic Mass Matrices
}

\author{
Alexander Kusenko用 and Robert Shrock ${ }^{\text {** }}$ \\ Institute for Theoretical Physics \\ State University of New York \\ Stony Brook, N. Y. 11794-3840
}

\begin{abstract}
We construct new invariants and give several theorems which determine in general (i) the number of physically meaningful phases in charged lepton and neutrino mass matrices and (ii) which elements of these matrices can be rendered real by rephasings. We illustrate our results with simple models.
\end{abstract}

*email: sasha@max.physics.sunysb.edu

** email: shrock@max.physics.sunysb.edu 
Understanding fermion masses and mixing remains one of the most important outstanding problems in particle physics. In particular, the issue of possible neutrino masses and associated lepton mixing is of fundamental interest. Although there is no definite direct evidence for nonzero neutrino masses they are expected on general grounds: given only the known left-handed neutrino fields and the usual Higgs field(s)[], nonzero neutrino masses result generically from higher-dimension operators which would occur at a scale near to that of quantum gravity, suppressed by associated inverse powers of the (reduced) Planck mass, $\bar{M}_{P}=\sqrt{\hbar c /\left(8 \pi G_{N}\right)}=2.4 \times 10^{18} \mathrm{GeV}$, For example, gauge-invariant dimension-5 operators of this type could produce neutrino masses of order $m_{\nu} \sim a v^{2} / \bar{M}_{P}$, where $v=250 \mathrm{GeV}$ is the scale of electroweak symmetry breaking, and $a$ is a dimensionless constant. Thus, one can understand on general grounds why neutrino masses are so small. If electroweak-singlet (EWS) neutrino fields exist, they would lead, via renormalizable, dimension-4 operators, to neutrino masses $m_{\nu} \sim v^{2} / M_{R}$, where the scale $M_{R}$ of the EWS neutrino mass is naturally $>>v$, again yielding, albeit for a different reason, very small $m_{\nu}$ [3]. In turn, a natural concomitant of (nondegenerate) neutrino masses is lepton mixing, which is thus also a generic expectation. It is thus of interest to study models for the leptonic mass matrices in the charge $Q=0$ and $Q=-1$ sectors. The diagonalization of these mass matrices determines both the masses and the observable lepton flavor mixing. In analyzing models of fermion masses and mixing, an important step is to determine the number of real amplitudes and unremovable, and hence physically meaningful, phases in the mass matrices. Surprisingly, there was no general solution in the literature to the problem of counting the number of complex phases and determining which elements of mass matrices can be rendered real by fermion rephasings. We recently presented a general solution for the quark sector [5]. The leptonic case is qualitatively different and more complicated because of the general presence of both Majorana and Dirac masses. In this Letter we give a solution for the leptonic case.

The mass terms for the charged leptons can be written in terms of the $G_{S M}=\mathrm{SU}(3) \times \mathrm{SU}(2)$ $\times \mathrm{U}(1)$ fields as

$$
-\mathcal{L}_{\text {mass }, Q=-1}=\sum_{j, k=1}^{3}\left[\bar{L}_{2 j L} M_{j k}^{(\ell)} \ell_{k R}\right]+\text { h.c. }
$$

where $j, k=1,2,3$ denote generation indices, $L_{j L}=\left(\begin{array}{l}\nu_{\ell_{j}} \\ \ell_{j}\end{array}\right)_{L}, \ell_{1}=e, \ell_{2}=\mu, \ell_{3}=\tau$, the first

\footnotetext{
${ }^{1}$ For reviews and current limits, see Refs. [n] and [2]. The apparent solar neutrino deficit is the most suggestive indirect evidence at present. The situation with atmospheric neutrinos is unclear.

${ }^{2}$ This comment applies formally to the standard model; however, the Higgs sector of this model is presumably stabilized by supersymmetry, in which there are (at least) a pair of Higgs fields with $Y=1$ and $Y=-1$.

${ }^{3}$ The lepton mixing angles are functions of ratios of elements of neutrino matrix elements and of charged lepton mass matrix elements, and even though left-handed neutrino masses are small, some of these ratios could, in principle, be $O(1)$. However, a set of conditions for natural suppression of observable lepton flavor violation were formulated, and it was shown that the standard model (generalized to include nonzero $m_{\nu}$ ) satisfies these [.
} 
subscript on $L_{a, j L}$ is the $\mathrm{SU}(2)$ index and $M^{(\ell)}$ is the charged lepton mass matrix.f In contrast to the charged lepton sector, where one at least knows the relevant fields, in the neutral lepton sector, one does not. In addition to the three known left-handed $I=1 / 2, I_{3}=1 / 2$ Weyl neutrino fields $\nu_{j L}, j=1-3$, there could be some number $n_{s}$ of electroweak-singlet neutrino fields $\chi_{j, R}$, $j=1,2, \ldots n_{s}$. The general neutrino mass matrix is given by

$$
-\mathcal{L}_{\text {mass }, Q=0}=\frac{1}{2}\left(\begin{array}{ll}
\bar{\nu}_{L} & \bar{\chi}_{L}^{c}
\end{array}\right)\left(\begin{array}{cc}
M^{(L)} & M^{(D)} \\
\left(M^{(D)}\right)^{T} & M^{(R)}
\end{array}\right)\left(\begin{array}{c}
\nu_{R}^{c} \\
\chi_{R}
\end{array}\right)+\text { h.c. }
$$

where $\nu_{L}=\left(\nu_{e}, \nu_{\mu}, \nu_{\tau}\right)_{L}^{T}, \chi_{R}=\left(\chi_{1}, \ldots, \chi_{n_{s}}\right)_{R}^{T} ; M_{L}$ and $M_{R}$ are $3 \times 3$ and $n_{s} \times n_{s}$ Majorana mass matrices, and $M_{D}$ is an 3 -row by $n_{s}$-column Dirac mass matrix We denote the $\left(3+n_{s}\right) \times\left(3+n_{s}\right)$ neutrino mass matrix in (2) as $M^{(0)}$. Thus, $M_{j k}^{(L)}=M_{j k}^{(0)}, j, k=1,2,3 ; \quad M_{j k}^{(R)}=M_{3+j, 3+k}^{(0)}$, $j, k=1, \ldots n_{s} ;$ and $M_{j k}^{(D)}=M_{3+j, k}^{(0)}, j=1,2,3, k=1, . ., n_{s}$, . In general, all of these matrices are complex. Recall that the anticommutativity of fermion fields in the path integral and the property $C^{T}=-C$ (where $C$ is the Dirac charge conjugation matrix) imply that

$$
M^{(f)}=M^{(f) T} \quad, \quad \text { for } f=L, R
$$

so that

$$
M^{(0)}=M^{(0) T}
$$

The diagonalization of (2) yields, in general, $3+n_{s}$ nondegenerate Majorana neutrino mass eigenstates (some could, of course, be degenerate in magnitude, leading to possible Dirac neutrino masses).

Although our theorems on phases are general, some comments are necessary concerning possible electroweak-singlet neutrinos. In the supersymmetric generalization of the standard model, which is strongly motivated by its ability to maintain the condition $v<<M_{P}$ beyond tree level without finetuning, the component fields $\chi_{j, L}^{c}$ would arise as part of $G_{S M}$-singlet chiral superfields $\hat{\chi}_{j}^{c}$ (all chiral superfields will be written as left-handed). One may recall that, in general, chiral superfields which are singlets under the standard model gauge group $G_{S M}$ can destabilize the hierarchy [6]. However, in commonly used models, the symmetry (such as matter parity) which prevents excessively rapid proton decay also excludes the types of terms which would destabilize the hierarchy.

To count the number of physically meaningful phases, we rephase the lepton fields so as to remove all possible phases in $M^{(\ell)}$ and $M^{(0)}$ by

$$
L_{j L}=e^{-i \alpha_{j}} L_{j L}^{\prime}
$$

\footnotetext{
${ }^{4}$ Here we use the result from LEP and SLC that there are three generations of fermions with associated light neutrinos [1].

${ }^{5}$ In the context of the (not necessarily minimal) supersymmetric standard model, we assume unbroken $R$ parity so that the neutrinos do not mix with the neutralinos (higgsinos and neutral color-singlet gauginos).
} 


$$
\ell_{j R}=e^{i \beta_{j}^{(\ell)}} \ell_{j R}^{\prime}
$$

for $j=1,2,3$, and, if there exist any $\chi_{j R}$ 's, also

$$
\chi_{j R}=e^{i \beta_{j}^{(\chi)}} \chi_{j R}^{\prime}
$$

for $j=1, \ldots n_{s}$. In terms of the primed (rephased) lepton fields, the mass matrices have elements

$$
M_{j k}^{(\ell) \prime}=e^{i\left(\alpha_{j}+\beta_{k}^{(\ell)}\right)} M_{j k}^{(\ell)}
$$

for the charged leptons, and, for the neutrino sector,

$$
M_{j k}^{(L) \prime}=e^{i\left(\alpha_{j}+\alpha_{k}\right)} M_{j k}^{(L)}
$$

for $j, k=1,2,3$;

$$
M_{j k}^{(R) \prime}=e^{i\left(\beta_{j}^{(\chi)}+\beta_{k}^{(\chi)}\right)} M_{j k}^{(R)}
$$

for $j, k=1, \ldots, n_{s}$, and

$$
M_{j k}^{(D) \prime}=e^{i\left(\alpha_{j}+\beta_{k}^{(\chi)}\right)} M_{j k}^{(D)}
$$

for $j=1,2,3$ and $k=1, \ldots, n_{s}$.

Thus, if $M^{(\ell)}$ has $N_{\ell}$ nonzero (and, in general, complex) elements, then the $N_{\ell}$ equations for making these elements real are

$$
\alpha_{j}+\beta_{k}^{(\ell)}=-\arg \left(M_{j k}^{(\ell)}\right)+\eta_{j k}^{(\ell)} \pi
$$

where the set $\{j k\}$ runs over each of these nonzero elements, and $\eta_{j k}^{(f)}=0$ or 1.9

Similarly, in the neutrino sector, if $M^{(D)}, M^{(L)}$, and $M^{(R)}$ have, respectively, $N_{D}, N_{L}$, and $N_{R}$ nonzero (and, in general, complex) elements, then the corresponding equations for making these elements real are

$$
\begin{gathered}
\alpha_{j}+\beta_{k}^{(\ell)}=-\arg \left(M_{j k}^{(D)}\right)+\eta_{j k}^{(D)} \pi \\
\alpha_{j}+\alpha_{k}=-\arg \left(M_{j k}^{(L)}\right)+\eta_{j k}^{(L)} \pi
\end{gathered}
$$

and

$$
\beta_{j}^{(\chi)}+\beta_{k}^{(\chi)}=-\arg \left(M_{j k}^{(R)}\right)+\eta_{j k}^{(R)} \pi
$$

where the various ranges of indices are obvious from (2). Let us define the $\left(6+n_{s}\right)$-dimensional vector of fermion field phases

$$
v=\left(\left\{\alpha_{i}\right\},\left\{\beta_{i}^{(\ell)}\right\},\left\{\beta_{i}^{(\chi)}\right\}\right)^{T}
$$

\footnotetext{
${ }^{6}$ The $\eta_{j k}$ term allows for the possibility of making the rephased element real and negative; this will not affect the counting of unremovable phases.
} 
where $\left\{\alpha_{i}\right\} \equiv\left\{\alpha_{1}, \alpha_{2}, \alpha_{3}\right\},\left\{\beta_{i}^{(\ell)}\right\} \equiv\left\{\beta_{1}^{(\ell)}, \beta_{2}^{(\ell)}, \beta_{3}^{(\ell)}\right\}$, and $\left\{\beta_{i}^{(\chi)}\right\} \equiv\left\{\beta_{1}^{(\chi)}, \ldots, \beta_{n_{s}}^{(\chi)}\right\}$. We also define the vector of phases of elements of the various mass matrices

$$
w=\left(\left\{\arg \left(M^{(\ell)}\right)+\eta_{j k}^{(\ell)} \pi\right\},\left\{\left\{\arg \left(M^{(0)}\right)+\eta_{j k}^{(0)} \pi\right\}\right)^{T}\right.
$$

of dimension equal to the number of rephasing equations $N_{e q}=N_{\ell}+N_{0}$, where $N_{0}=N_{L}+N_{R}+N_{D}$. We can then write (12)- (15) as

$$
T v=w
$$

which defines the $N_{e q}$-row by $\left(6+n_{s}\right)$-column matrix $T$.

Our first main theorem is: The number of unremovable and hence physically meaningful phases $N_{p}$ in $M^{(\ell)}$ and $M^{(0)}$ is

$$
N_{p}=N_{e q}-\operatorname{rank}(T)
$$

In order for these phases to be physically meaningful, it is, of course, necessary that they be unchanged under the full set of rephasings of fermion fields. In fact, as we will show, there is a oneto-one correspondence between each such unremovable phase and an independent phase of a certain product of elements of mass matrices which is invariant under fermion field rephasings. (In special cases a model may have an unremovable, invariant phase which, because of a particular symmetry, is zero or $\pi$; we give an example below.) The proof of (19) is similar to that for the analogous theorem which we proved for the quark sector [5]: Let $\operatorname{rank}(T)=r_{T}$. Then one can delete $N_{e q}-r_{T}$ rows from the matrix $T$, i.e. not attempt to remove the phases from the corresponding elements of the $M^{(\ell)}$ and $M^{(0)}$. For the remaining $r_{T}$ equations, one moves a subset of $6+n_{s}-\left(N_{e q}-r_{T}\right)$ phases in $v$ to the right-hand sides of (12)-(15), thus including them in a redefined $\bar{w}$. This yields a set of $r_{T}$ linear equations in $r_{T}$ unknown phases, denoted $\bar{v}$. We write this as $\bar{T} \bar{v}=\bar{w}$. Since, by construction, $\operatorname{rank}(\bar{T})=r_{T}, \bar{T}$ is invertible, so that one can now solve for the $r_{T}$ fermion rephasings in $\bar{v}$ which render $r_{T}$ of the $N_{e q}$ complex elements real. Hence there are $N_{e q}-r_{T}$ remaining phases in $M^{(\ell)}$ and $M^{(0)}$, as claimed.

Some comments are in order. First, as is clear from our proof, in general, the result (19) does not depend on whether or not $M_{j k}^{(f)}=M_{k j}^{(f)}$ initially for $f=\ell, D$. Hence making $M^{(\ell)}$ or $M^{(D)}$ initially (complex) symmetric does not, in general, result in any reduction in $N_{p}$, since in terms of the $G_{S M}$ theory, nothing guarantees this symmetry, and the rephasing is carried out in terms of $G_{S M}$ fields. Second, if one of the unremovable phases is put in a given off-diagonal $M_{p q}^{(f)}$ for $f=\ell$

\footnotetext{
${ }^{7}$ In the quark case, $T$ is a $N_{e q} \times 9$ matrix, and we noted 7 that it could not have maximal rank because one overall rephasing left the Yukawa (or equivalently, mass) matrices invariant. In the present case, unless $M^{(L)}$ and $M^{(R)}$ both vanish, there is no analogous overall rephasing which leaves $M^{(\ell)}$ and $M^{(0)}$ invariant, and hence $T$ can have maximal rank.
} 
or $D$, one may wish to modify the $q p^{\prime}$ th equation to read

$$
\alpha_{q}+\beta_{p}^{(f)}=-\arg \left(M_{q p}^{(f)}\right)-\arg \left(M_{p q}^{(f)}\right)
$$

for $f=\ell, D$. For example, in a model where $\left|M_{p q}^{(f)}\right|=\left|M_{q p}^{(f)}\right|$, this would yield $M_{p q}^{(f) *}=M_{q p}^{(f)}$ for this pair $p q$ and $f=\ell$ or $D$. The modification in (20) has no effect on the counting of phases. Of course, given the property (3), when one rephases to make $M_{j k}^{(f)}$ real for $f=L, R$, this automatically does the same for $M_{k j}^{(f)}$.

A fundamental question concerns which elements of $M^{(\ell)}$ and $M^{(0)}$ (taken to be complex initially) can be made real by fermion rephasings. This is connected with the issue of which rows are to be removed from $T$ to obtain $\bar{T}$, i.e. which nonzero elements of $M^{(\ell)}$ and $M^{(0)}$ are left complex. We present some more theorems which answer this question. The general method is to construct all independent complex products of elements of $M^{(\ell)}$ and $M^{(0)}$ which are rephasing-invariant with respect to (5), (6) and, if $\chi_{j R}$ 's are present, also (7). These must involve an even number of such elements, since for each index on an $M$, there must be a corresponding index on an $M^{*}$ in order to form a rephasing-invariant product. Since in general, by construction, these have arguments $\neq 0, \pi$, each one implies a constraint which is that the set of $2 n$ elements which comprise it cannot be made simultaneously real by any rephasings or the charged lepton or neutrino fields. (In special cases, a symmetry of a model may render some of these real,.) In the neutrino sector, although the Dirac mass matrix conserves the global lepton number $\mathrm{U}(1)$, while the Majorana mass matrices violate this symmetry, it is possible and convenient to treat $\nu_{j R}^{c}$ and $\chi_{k R}$ together, as was done in (2), the RHS of which can be written simply as $(1 / 2) \bar{f}_{L}^{(0)} M^{(0)} f_{R}^{(0)}+$ h.c.. The rephasings in (5) and (7) can be represented in a similarly unified way as $f_{j R} \rightarrow e^{i \gamma_{j}} f_{j R}$. We thus construct the rephasing invariants

$$
P_{2 n ; j_{1} k_{1}, \ldots j_{n} k_{n} ; \sigma_{L}}^{(\ell)}=\prod_{a=1}^{n} M_{j_{a} k_{a}}^{(\ell)} M_{\sigma_{L}\left(j_{a}\right) k_{a}}^{(\ell) *}
$$

where $f=\ell$ and $\sigma_{L}$ is an element of the permutation group $S_{n}$, and

$$
P_{2 n ; j_{1} j_{2}, \ldots, j_{2 n-1} j_{2 n} ; \sigma}^{(0)}=\prod_{a=1}^{n} M_{j_{2 a-1} j_{2 a}}^{(0)} M_{\sigma\left(j_{2 a-1}\right) \sigma\left(j_{2 a}\right)}^{(0) *}
$$

where $\sigma \in S_{2 n}$. (The difference in structure is due to (4).)

At quartic order, $2 n=4$, (21) and (22) both yield the same complex invariant, which (in the notation of (21)) is

$$
P_{4 ; j_{1} k_{1}, j_{2} k_{2} ; \tau}^{(f)} \equiv P_{j_{1} k_{1}, j_{2} k_{2}}^{(f)}=M_{j_{1} k_{1}}^{(f)} M_{j_{2} k_{2}}^{(f)} M_{j_{2} k_{1}}^{(f) *} M_{j_{1} k_{2}}^{(f) *}
$$

for $f=\ell, 0$. Note the symmetries

$$
P_{j_{1} k_{1}, j_{2} k_{2}}^{(f)}=P_{j_{2} k_{2}, j_{1} k_{1}}^{(f)}
$$




$$
P_{j_{1} k_{1} j_{2} k_{2}}^{(f)}=P_{j_{1} k_{2}, j_{2} k_{1}}^{(f) *}
$$

(whence also $P_{j_{1} k_{1}, j_{2} k_{2}}^{(f)}=P_{j_{2} k_{1}, j_{1} k_{2}}^{(f) *}$ ) for $f=\ell, 0$ and, for $f=0$, the additional symmetry

$$
P_{j_{1} j_{2}, j_{3} j_{4}}^{(0)}=P_{j_{2} j_{1}, j_{4} j_{3}}^{(0)}
$$

In a shorthand notation, (23) for $f=\ell$ may be denoted $\ell \ell \ell^{*} \ell^{*}$. It is useful to classify the complex $P^{(0)}$ invariants according to which submatrices $-M^{(L)}, M^{(R)}$, and/or $M^{(D)}-$ in $M^{(0)}$ they involve. We find five possible (complex) structures: $L L L^{*} L^{*}, R R R^{*} R^{*}, L D L^{*} D^{*}, R D R^{*} D^{*}$, and $L R D^{*} D^{*}$. Explicitly, the first two are given by (23) for $f=L, R$, respectively, and the others by the products

$$
\Pi_{j_{1} j_{2}, j_{3} m_{1}}^{(L f)}=M_{j_{1} j_{2}}^{(L)} M_{j_{3} m_{1}}^{(f)} M_{j_{1} j_{3}}^{(L) *} M_{j_{2} m_{1}}^{(f n n) *}
$$

for $f=D$,

$$
\Xi_{k_{1} k_{2}, j_{1} k_{3}}^{(R D)}=M_{k_{1} k_{2}}^{(R)} M_{j_{1} k_{3}}^{(D)} M_{k_{1} k_{3}}^{(R) *} M_{j_{1} k_{2}}^{(D) *}
$$

and

$$
\Omega_{j_{1} j_{2}, k_{1} k_{2}}^{(L R D D)}=M_{j_{1} j_{2}}^{(L)} M_{k_{1} k_{2}}^{(R)} M_{j_{1} k_{1}}^{(D) *} M_{j_{2} k_{2}}^{(D) *}
$$

In terms of $P^{(0)}$ 's (and using (4), these are

$$
\begin{gathered}
\Pi_{j_{1} j_{2}, j_{3} m_{1}}^{(L D)}=M_{j_{1}, j_{2}}^{(0)} M_{j_{3}, 3+m_{1}}^{(0)} M_{j_{1}, j_{3}}^{(0) *} M_{j_{2}, 3+m_{1}}^{(0) *}=P_{j_{2} j_{1}, j_{3} 3+m_{1}}^{(0)} \\
\Xi_{k_{1} k_{2}, j_{1} k_{3}}^{(R D)}=M_{3+k_{1}, 3+k_{2}}^{(0)} M_{j_{1}, 3+k_{3}}^{(0)} M_{3+k_{1}, 3+k_{3}}^{(0) *} M_{j_{1}, 3+k_{2}}^{(0) *}=P_{3+k_{1} 3+k_{2}, j_{1} 3+k_{3}}^{(0)} \\
\Omega_{j_{1} j_{2}, k_{1} k_{2}}^{(L R D D}=M_{j_{1}, j_{2}}^{(0)} M_{3+k_{1}, 3+k_{2}}^{(0)} M_{j_{1}, 3+k_{1}}^{(0) *} M_{j_{2}, 3+k_{2}}^{(0) *}=P_{j_{2} j_{1}, 3+k_{1} 3+k_{2}}^{(0)}
\end{gathered}
$$

Secondly, we construct a set of complex invariants connecting the $Q=-1$ and $Q=0$ lepton sectors. At order $2 n=4$ we find two independent types of complex invariants, which we denote as

$$
Q_{j_{1} k_{1}, j_{2} m_{1}}^{(D \ell)}=M_{j_{1} k_{1}}^{(D)} M_{j_{2} m_{1}}^{(\ell)} M_{j_{2} k_{1}}^{(D) *} M_{j_{1} m_{1}}^{(\ell) *}
$$

and $\Pi_{j_{1} j_{2}, j_{3} m_{1}}^{L \ell}$ as given by (27) for $f=\ell$. Note that

$$
Q_{j_{1} k_{1}, j_{2} m_{1}}^{(D \ell)}=Q_{j_{2} k_{1}, j_{1} m_{1}}^{(D \ell) *}
$$

In the short notation, these are $D \ell D^{*} \ell^{*}$ and $L \ell L^{*} \ell^{*}$. We also observe that

$$
\Pi_{j_{1} j_{2}, j_{3} m_{1}}^{(L f)}=Q_{j_{2} j_{1}, j_{3} m_{1}}^{(L, f)}
$$

At order $2 n=6$, we find first the complex invariants $f f f f^{*} f^{*} f^{*}$ for $f=\ell, 0$, each of which involves only one of the two charge sectors. The various possibilities for the permutations in (21) and (22) yield only one independent explicit form, which we denote simply by

$$
P_{j_{1} k_{1}, j_{2} k_{2}, j_{3} k_{3}}^{(f)}=M_{j_{1} k_{1}}^{(f)} M_{j_{2} k_{2}}^{(f)} M_{j_{3} k_{3}}^{(f)} M_{j_{2} k_{1}}^{(f) *} M_{j_{3} k_{2}}^{(f) *} M_{j_{1} k_{3}}^{(f) *}
$$


The $f=\ell$ invariant is denoted $\ell \ell \ell \ell^{*} \ell^{*} \ell^{*}$. The $f=0$ invariants can be further characterized according to which submatrices of $M^{(0)}$ they involve; we find the following types: $L L L L^{*} L^{*} L^{*}$, $R R R R^{*} R^{*} R^{*}, D D D D^{*} D^{*} D^{*}, D D L D^{*} D^{*} L^{*}, D D R D^{*} D^{*} R^{*}, L L D L^{*} L^{*} D^{*}, R R D R^{*} R^{*} D^{*}$, $L R D L^{*} R^{*} D^{*}, L L R L^{*} D^{*} D^{*}, L R R D^{*} D^{*} R^{*}$, and $L R D D^{*} D^{*} D^{*}$. (In our notation, $f f f^{\prime} f^{*} f^{*} f^{*}$ and $f^{\prime} f f f^{\prime *} f^{*} f^{*}$ are synonymous; also, $L D D L^{*} L^{*} R^{*}$ contains the same phase information as $L L R L^{*} D^{*} D^{*}$, and so forth for the other conjugates of this type, which are hence not listed.) For any $f$, the invariants in $(36)$ satisfy

$$
P_{j_{1} k_{1}, j_{2} k_{2}, j_{3} k_{3}}^{(f)}=P_{j_{2} k_{2}, j_{3} k_{3}, j_{1} k_{1}}^{(f)}=P_{j_{3} k_{3}, j_{1} k_{1}, j_{2} k_{2}}^{(f)}
$$

and

$$
P_{j_{1} k_{1}, j_{2} k_{2}, j_{3} k_{3}}^{(f)}=P_{j_{3} k_{2}, j_{2} k_{1}, j_{1} k_{3}}^{(f) *}
$$

For the cases where $M^{(f)}$ is symmetric, i.e. $M^{(f)}=M^{(0)}$ or, for submatrices, $M^{(L)}, M^{(R)}$, we find the additional symmetry

$$
P_{j_{1} j_{2}, j_{3} j_{4}, j_{5} j_{6}}^{(f)}=P_{j_{2} j_{1}, j_{6} j_{5}, j_{4} j_{3}}^{(f)}
$$

We also find the following independent 6 'th order complex invariants linking the charge $Q=-1$ and $Q=0$ sectors: $\ell \ell L \ell^{*} \ell^{*} L^{*}, L L \ell L^{*} L^{*} \ell^{*}, \ell \ell D \ell^{*} \ell^{*} D^{*}, D D \ell D^{*} D^{*} \ell^{*}, \ell D L \ell^{*} D^{*} L^{*}, \ell D R \ell^{*} D^{*} R^{*}$, $\ell L R \ell^{*} D^{*} D^{*}$. The 6'th order mixed-charge invariants of the form $f f f^{\prime} f^{*} f^{*} f^{*^{\prime}}$, and also the 6 'th order $Q=0$ invariants which are of this form when written in terms of $L, R$ and/or $D$ submatrices, are given explicitly as

$$
Q_{j_{1} k_{1}, j_{2} k_{2}, j_{3} m_{1}}^{\left(f f f^{\prime}\right.}=M_{j_{1} k_{1}}^{(f)} M_{j_{2} k_{2}}^{(f)} M_{j_{3} m_{1}}^{\left(f^{\prime}\right)} M_{j_{1} k_{2}}^{(f) *} M_{j_{3} k_{1}}^{(f) *} M_{j_{2} m_{1}}^{\left(f^{\prime}\right) *}
$$

Some examples of explicit forms of other invariants include

$$
\begin{aligned}
\ell D R \ell^{*} D^{*} R^{*}: & M_{j_{1} k_{1}}^{(\ell)} M_{j_{2} m_{1}}^{(D)} M_{m_{2} m_{3}}^{(R)} M_{j_{2} k_{1}}^{(\ell) *} M_{j_{1} m_{2}}^{(D) *} M_{m_{1} m_{3}}^{(R) *} \\
\ell L R \ell^{*} D^{*} D^{*}: & M_{j_{1} k_{1}}^{(\ell)} M_{j_{2} j_{3}}^{(L)} M_{m_{1} m_{2}}^{(R)} M_{j_{2} k_{1}}^{(\ell) *} M_{j_{1} m_{1}}^{(D) *} M_{j_{3} m_{2}}^{(D) *} \\
\ell D L \ell^{*} D^{*} L^{*}: \quad & M_{j_{1} k_{1}}^{(\ell)} M_{j_{2} m_{1}}^{(D)} M_{j_{3} j_{4}}^{(L)} M_{j_{2} k_{1}}^{(\ell) *} M_{j_{3} m_{1}}^{(D) *} M_{j_{1} j_{4}}^{(L) *} \\
& M_{j_{1} k_{1}}^{(\ell)} M_{j_{2} m_{1}}^{(D)} M_{j_{3} j_{4}}^{(L)} M_{j_{3} k_{1}}^{(\ell) *} M_{j_{4} m_{1}}^{(D) *} M_{j_{1} j_{2}}^{(L) *}, \quad \text { etc. }
\end{aligned}
$$

Further discussion of higher order invariants is presented elsewhere [7].

Our theorems are as follows: For a given model, construct the maximal set of independent generally complex invariants; denote the number of these as $N_{i n v}$. Of the corresponding $N_{i n v}$ arguments, a subset $N_{i a}$ are linearly independent. Then (a) the number of unremovable, invariant, and hence physically meaningful phases $N_{p}$ is equal to $N_{i a}$; (b) in the general case in which the $N_{i a}$ 
arguments are $\neq 0, \pi$, each implies a constraint that elements contained within the corresponding invariant cannot all be made simultaneously real; (b) this constitutes the complete set of constraints on which elements of $M^{(\ell)}$ and $M^{(0)}$ can be made simultaneously real. Often, the lowest-order nonvanishing complex invariants are quartic, but we give an exception below.

In order to determine $N_{i a}$ from the $N_{i n v}$ independent complex invariants, we proceed as follows. Form the $N_{e q}$ vector of arguments of nonzero (and in general complex) elements of $M^{(\ell)}$ and $M^{(0)}, \xi$. For each complex invariant of a given order, $X, \arg (X)=\sum_{f} \sum_{j, k} c_{j, k}^{(f)} \arg \left(M^{(f)}\right)_{j k}$, where the sum is over the $N_{e q}$ nonzero elements of $M^{(\ell)}$ and $M^{(0)}$. These equations can be written as $Z \xi=w$, where $\xi$ is the $N_{i n v}$-dimensional vector $\xi=\left(\arg \left(X_{1}\right), \ldots, \arg \left(X_{N_{i n v}}\right)\right)$ and $Z$ is an $N_{i n v}$-row by $N_{e q}$-column matrix. Then $\operatorname{rank}(Z)=N_{i a}$.

We illustrate our general theorems with some models.8 We first consider the case $n_{s}=0$, i.e. no electroweak-singlet neutrinos. For models of this type, $v$ is 6 -dimensional, and $T$ is $N_{e q} \times 6$ dimensional. As an illustration, consider a model defined by

$$
\begin{aligned}
& M^{(\ell)}=\left(\begin{array}{ccc}
0 & E_{12} & 0 \\
E_{21} & E_{22} & E_{23} \\
0 & E_{32} & E_{33}
\end{array}\right) \\
& M^{(L)}=\left(\begin{array}{ccc}
0 & L_{12} & 0 \\
L_{12} & L_{22} & L_{23} \\
0 & L_{23} & L_{33}
\end{array}\right)
\end{aligned}
$$

(We have verified that this model is able to fit current solar neutrino data and other established limits on neutrino masses and mixing.) For this model, there are $N_{e q}=10$ complex elements in $M^{(\ell)}$ and $M^{(D)}$ and corresponding rephasing equations. The matrix $T$ is $10 \times 6$, and we find that it has rank 6. Our theorem (19) then implies that there are $N_{p}=N_{e q}-\operatorname{rank}(T)=4$ unremovable phases in $M^{(\ell)}$ and $M^{(L)}$. We find $N_{i n v}=8$ independent complex (quartic) invariants: $P_{22,33}^{(\ell)}=$ $E_{22} E_{33} E_{32}^{*} E_{23}^{*}, P_{22,33}^{(0)}=P_{22,33}^{(L)}=L_{22} L_{33} L_{23}^{* 2}, \Pi_{12,22}^{(L \ell)}=L_{12} E_{22} L_{22}^{*} E_{12}^{*}, \Pi_{12,32}^{(L \ell)}=L_{12} E_{32} L_{23}^{*} E_{12}^{*}$ $\Pi_{22,32}^{(L \ell)}=L_{22} E_{32} L_{23}^{*} E_{22}^{*} \Pi_{22,33}^{(L \ell)}=L_{22} E_{33} L_{23}^{*} E_{23}^{*}, \Pi_{23,32}^{(L \ell)}=L_{23} E_{32} L_{33}^{*} E_{22}^{*}, \Pi_{23,33}^{(L \ell)}=L_{23} E_{33} L_{33}^{*} E_{23}^{*}$. From these we calculate the $8 \times 10$ dimensional $Z$ matrix and find that it has rank 4 , so that of the eight arguments of the complex invariants, there are $N_{i a}=4$ linearly independent ones, in accord with the equality $N_{i a}=N_{p}$ and our result that $N_{p}=4$. Thus, for this model, it is not, in general, possible by any rephasings to make either the charged lepton or neutrino mass matrices real. Furthermore, the last complex invariant implies that it is not possible in general to make the

\footnotetext{
${ }^{8}$ Since our purpose is to illustrate our general theorems, we do not attempt to discuss an exhaustive set of viable models of neutrino masses and mixing. Indeed, given the unsettled status of atmospheric neutrino oscillations (claimed by some experiments, not by others), it is unclear what data a viable model must fit. Here we shall tentatively assume that the solar neutrino deficit does indicate neutrino oscillations but shall view it as premature to make any inference of new physics from the atmospheric neutrino oscillation data.
} 
set of elements $\left\{L_{23}, L_{33}, E_{23}, E_{33}\right\}$ simultaneously real; the next-to-last implies the same for the set $\left\{L_{23}, L_{33}, E_{22}, E_{32}\right\}$, and so forth for each of the other complex invariants.

A natural setting for models with electroweak-singlet neutrinos is an $\mathrm{SO}(10)$ grand unified theory (GUT), where a $\chi_{j L}^{c}$ occurs, along with the SU(5) $\mathbf{1 0}_{L}$ and $\overline{\mathbf{5}}_{L}$ in the $\mathrm{SO}(10) \mathbf{1 6}_{L}$, for each of the three generations, so that $n_{s}=3$. An interesting model of this type was recently studied in a supersymmetric context in Ref. [8] (DHR). A pioneering study of a non-supersymmetric $\mathrm{SO}(10)$ model which was similar but had more mass matrix parameters was carried out in Ref. [9]. In both cases the Higgs representations were restricted so as to obtain (complex) symmetric mass matrices. Here we shall consider two models. First, to exhibit the generality of our results, we will consider a generalization of the models of Refs. [8] and $\mathrm{r}$ in which $M^{(\ell)}$ and $M^{(D)}$ are not assumed to be symmetric (recall from (3) that $M^{(R)}$ is automatically symmetric)

$$
\begin{aligned}
M^{(\ell)} & =\left(\begin{array}{ccc}
0 & E_{12} & 0 \\
E_{21} & E_{22} & 0 \\
0 & 0 & E_{33}
\end{array}\right) \\
M^{(D)} & =\left(\begin{array}{ccc}
0 & D_{12} & 0 \\
D_{21} & 0 & D_{23} \\
0 & D_{32} & D_{33}
\end{array}\right) \\
M^{(R)} & =\left(\begin{array}{ccc}
0 & R_{12} & 0 \\
R_{12} & 0 & 0 \\
0 & 0 & R_{33}
\end{array}\right)
\end{aligned}
$$

(Here all entries are in general complex.) Second, we shall consider the DHR model. We note that, to reduce the number of parameters, the Yukawa couplings were further restricted in the DHR model so that (besides the conditions $M^{(\ell)}=M^{(\ell) T}$ and $\left.M^{(D)}=M^{(D) T}\right) D_{12}=-3 R_{12}$ and $D_{33}=-3 R_{33}$. For the general model (46)-(48) we calculate the $11 \times 9$ dimensional $T$ matrix and find that it has rank 9 , so that by our theorem (19) there are $N_{p}=N_{e q}-\operatorname{rank}(T)=2$ unremovable, physically meaningful phases in the leptonic sector. Correspondingly, we find two independent complex invariants (which have independent arguments), so $N_{i n v}=N_{i a}=2$. These are both of 6 'th order: $P_{24,53,66}^{(0)}=D_{21} D_{32} R_{33} D_{23}^{*} D_{33}^{*} R_{12}^{*}$ and $Q_{32,23,12}^{(D D \ell)}=D_{32} D_{23} E_{12} D_{12}^{*} D_{33}^{*} E_{22}^{*}$. As can be checked explicitly here, making $M^{(\ell)}$ and $M^{(D)}$ (complex) symmetric does not change the value of $N_{p}$ or make either of the complex invariants real. For the general model (and for the special case in which $M^{(\ell)}$ and $M^{(D)}$ are symmetric), one thus has the constraints that the elements in the set $\left\{D_{21}, D_{23}, D_{32}, D_{33}, R_{12}, R_{33}\right\}$ cannot be made simultaneously real by any fermion rephasing, and similarly, the elements in the set $\left\{D_{12}, D_{23}, D_{32}, D_{33}, E_{12}, E_{22}\right\}$ cannot be made simultaneously real by any rephasings; the first constraint shows that it is not possible to make both the Dirac neutrino mass matrix $M^{(D)}$ and the right-handed Majorana mass matrix $M^{(R)}$ simultaneously real 
by any fermion field rephasings. For the light particle sector of the general model, our constraints allow one to place (i) both phases in the light neutrino mass matrix $M_{\nu}=M^{(D)} M^{(R)-1} M^{(D) T}$ (with $M^{(\ell)}$ being made real); or (ii) one phase in $M_{\nu}$ and one in $M^{(\ell)}$; but (iii) not both in $M^{(\ell)}$. In contrast, for the DHR model, it was noted [8] that there is only one (complex) phase in the leptonic sector (and indeed in the model as a whole). In terms of our formalism, this follows because, as a result of the relation between $M^{(D)}$ and $M^{(R)}$ given above, $P_{24,53,66}^{(0)}$ is actually real, so that its phase, while still an unremovable, invariant, and hence meaningful phase, is no longer complex. It is still technically true that $N_{p}=N_{i a}=2$ in this model, but only one of these phases is complex. This model (in both its general form and the above specializations) also serves as an example of one in which there are no complex quartic invariants. We have shown that this is true of the quark sector of the model, where we found [5] one 6'th order invariant, which, written in terms of Yukawa matrix elements, is $Q_{32,23,12}^{(u u d)}=Y_{32}^{(u)} Y_{23}^{(u)} Y_{12}^{(d)} Y_{12}^{(u) *} Y_{33}^{(u) *} Y_{22}^{(d) *}$. Đ Further results and applications will be reported elsewhere [7].

This research was supported in part by NSF grant PHY-93-09888.

\section{References}

[1] Particle Data Group, Review of Particle Properties, 1992 Edition Phys. Rev. D45II (1992); 1994 Edition, in preparation.

[2] A. Smirnov, review talk in the 1993 International Lepton Photon Symposium, Cornell, Aug. $1993 ;$ hep-ph/9310368.

[3] M. Gell-Mann, R. Slansky, and P. Ramond, in Supergravity, eds. D. Freedman and P. van Nieuwenhuizen (North Holland, 1979), p. 315; T. Yanagida, Prog. Theor. Phys. B135 (1978) 66; Proc. Workshop on Unified Theory and Baryon Number in the Universe (KEK, Japan, 1979).

[4] B. W. Lee and R. Shrock, Phys. Rev. D16 (1977) 1444.

[5] A. Kusenko and R. Shrock, ITP-SB-93-58 (hep-ph/9310307).

[6] H. P. Nilles, M. Srednicki, D. Wyler, Phys. Lett. B124 (1983) 337; A. Lahanas, Phys. Lett. B124 (1983) 341; H. P. Nilles, Phys. Repts. 110 (1984) 1; J. Kubo and S. Sakakibara, Phys. Lett. B140 (1984); J. Bagger and E. Poppitz, Phys. Rev. Lett.71 (1993) 2380 hepph/9307317).

\footnotetext{
${ }^{9}$ Note that in the models of [8] and [9], $\arg \left(Q_{32,23,12}^{(u u d)}\right)=\arg \left(Q_{32,23,12}^{(D D \ell)}\right)-\pi$ so that this does not represent a new phase in the theory as a whole.
} 
[7] A. Kusenko and R. Shrock, ITP-SB-93-68, to appear.

[8] S. Dimopoulos, L. Hall, and S. Raby, Phys. Rev. D47 (1993) R3697 (hep-ph/9208243). We thank L. Hall and S. Raby for a discussion of this model.

[9] J. Harvey, D. Reiss, and P. Ramond, Nucl. Phys. B199 (1982) 223. 